\title{
Value of Digital Breast Tomosynthesis versus Additional Views for the Assessment of Screen-Detected Abnormalities - a First Analysis
}

\author{
Sylvia Heywang-Köbrunner ${ }^{a, b} \quad$ Alexander Jaensch ${ }^{a, b} \quad$ Astrid Hacker ${ }^{a} \quad$ Sabina Wulz-Horber ${ }^{b}$ \\ Thomas Mertelmeier ${ }^{c}$ Dieter Hölzel ${ }^{d}$ \\ ${ }^{a}$ National Reference Centre Mammography Munich, Munich, Germany; \\ ${ }^{\mathrm{b}}$ Brustdiagnostik München, Munich, Germany; \\ ' Siemens Healthcare $\mathrm{GmbH}$, Erlangen, Germany; \\ ${ }^{d}$ Em. Head of IBE Institute of Biomathematics and Epidemiology, Clinical Cancer Registry, Munich, Germany
}

\section{Keywords}

Breast imaging · Digital breast tomosynthesis . Assessment $\cdot$ Screening

\section{Summary}

Background: The purpose of this study was to countercheck the equivalence of single-view digital breast tomosynthesis (DBT) or DBT with additional views (DBT+AV) compared to traditional standard assessment by additional views (AV) in patients with a screen-detected abnormality. Patients and Methods: Patients with a screen-detected abnormality were consecutively invited to obtain 1 single-view wide-angle DBT in addition to the indicated AV. The study was approved by the local ethics committee and by the Federal Office for Radiation Protection. Results: This study is based on 311 lesions in 285 patients with a follow-up of $>2$ years and/or biopsy. Counting BI-RADS 0 and 3 as positive calls, the sensitivity/specificity of DBT+AV versus DBT only versus AV only were $96.4 / 54.3 \%, 96.4 / 56.6 \%$, and $90.9 / 42.2 \%$, respectively. The specificities and BI-RADS classifications differed significantly $(p<0.01$ ). AV appeared unnecessary in $88.8 \%$ of the cases. Conclusion: DBT appeared to be at least equivalent to $\mathrm{AV}$ for assessing indeterminate screen-detected lesions and could replace AV for most lesions. To obtain the extra information appears possible without increasing the overall radiation dose. Subsequent blinded reader studies are ongoing.

(C) 2017 S. Karger GmbH, Freiburg

\section{KARGER}

() 2017 S. Karger GmbH, Freiburg

\section{Introduction}

Assessment of screen-detected abnormalities remains a demanding task and in the majority of cases requires the use of additional mammographic views (AV).

Currently, the traditional imaging assessment (mostly based on mammographic AV and ultrasound) is able to decrease false-positive calls but cannot eliminate them. False-negative imaging assessment results (classification or perception errors) are known to occur in a limited number of cases $[1,2]$. Digital breast tomosynthesis (DBT) has become available as a promising further development of digital mammography. Its additional or substitutive application is hoped to further increase the accuracy of imaging assessment.

First experiences with the application of DBT in a diagnostic setting have been encouraging [3-6]. However, only few authors have so far directly compared the information obtained from AV or standard assessment with that from DBT on consecutive screendetected lesions [7-10], some with low numbers [7, 8]. Also data with long-term follow-up of benign diagnoses are limited. Since the imaging technology can vary between different DBT models, studies to compare the different technologies are desirable.

The purpose of this study was to investigate the value of wideangle DBT for the assessment of screen-detected lesions. Testing based on screen-detected abnormalities was chosen for the following reasons:

Firstly, according to our experience, the number of subtle and demanding lesions is highest within a screening population, which by definition predominantly consists of clinically asymptomatic women. Secondly, offering DBT to consecutive patients with a defined screen-detected abnormality promises to reduce selection bias and to allow assessment of the new method in a defined setting. 
The specific goals of this study were: i) to compare the diagnostic accuracy (sensitivity, specificity) of traditional standard assessment (using AV only) ('AV') to tomosynthesis alone ('DBT') and to the combination of $\mathrm{AV}$ and tomosynthesis ('AV+DBT'); ii) to assess the need for AV in women undergoing DBT; and iii) to select diagnostic situations where DBT may or may not be helpful.

This paper presents a first evaluation of the results as obtained directly during the study and as counterchecked by consensus in retrospect.

\section{Patients and Methods}

\section{Study Protocol}

The study protocol was approved by the ethics committee of the Bavarian Medical Association and by the Federal Office for Radiation Protection. All patients gave informed consent.

Single-view tomosynthesis was consecutively offered to patients aged 50-69 years with a screen-detected abnormality that necessitated further mammographic assessment by mammographic AV.

All participating patients underwent routine diagnostic work-up with mostly 1-2 mammographic AV. Furthermore, they underwent 1 single-view DBT. Ultrasound was also used following our usual assessment standard. This included bilateral whole-breast ultrasound for all assessment cases with an American College of Radiology (ACR)2-4 tissue density, and assessment of all lesions except microcalcifications of the involved breast only for ACR1 tissue density. Decisions concerning further work-up of an individual patient were always based on all available knowledge according to good clinical practice.

The study diagnoses only concerned information from 'AV', 'DBT', or 'DBT+AV'. Diagnoses were recorded using the BI-RADS categories, as well as information on lesion type, breast density, and number and type of AV. If information by ultrasound or (rarely) magnetic resonance imaging (MRI) was considered essential for the final diagnosis, the mammographic diagnosis (with or without DBT) was classified as BI-RADS 0. If short-term follow-up was considered necessary, the diagnosis was classified as BI-RADS 3. The study diagnoses for each method were based on the method tested (AV or DBT or AV+DBT) and on the initial screening mammogram, and may thus not always correspond with the diagnosis based on the complete imaging assessment. The initial study design included the recording of the prospective diagnoses only and the comparative evaluation of the diagnostic accuracy based on histopathological proof and 2-year follow-up.

In order to increase objectiveness, a second reading and consensus were included.

The diagnoses in this publication are based on a consensus reading of 2 readers: The diagnoses of Reader 1 (the principal investigator) were made prospectively during the study and thus blinded to the final result (based on biopsy, further methods, or follow-up). Prospective blinding between the study methods was not possible since correct decisions during and at the end of the imaging assessment required a combined evaluation of the available information. The diagnoses of Reader 2 were made based on a retrospective reading of the anonymized images of the test method and the screening mammogram. The reading took place 3 years after the study. Reader 2 was also blinded to the final diagnoses and to information from other methods (ultrasound or MRI), as well as to the diagnoses of the first reader.

Being aware of potential bias due to absent blinding between the mammographic methods, both readers paid special attention to not use a different BIRADS classification for the different combinations of methods unless clearly different information was presented. Discrepancies underwent a retrospective consensus reading. This was performed in a separate session after reading 2, again blinded to the final result and to other methods.

All suspicious findings (BI-RADS 4 or 5) were verified by histopathology (and follow-up). Unsuspicious findings (BI-RADS 1-3) were verified by assessment based on all methods (including ultrasound and if needed MRI) and by follow-up of $>2$ years. Of a total of 311 lesions, 111 lesions were proven by histopathology; another 161 cases were proven by at least 2 years of mammographic follow-up; for a further 19 lesions, clinical or sonographic follow-up of $>2$ years is available. Cancer registry matching for the remaining 20 cases with a benign diagnosis yielded no additional case of breast cancer.

The diagnoses of the consensus were classified as true-positive, false-negative, true-negative, or false-positive with respect to the final diagnosis (as verified by $03 / 2016$ ).

\section{Patients and Lesions}

Recruitment started in May 2010 and stopped in November 2011. Altogether, 284 patients with 311 lesions were evaluated. All further evaluations will be reported lesion-based.

\section{Evaluation}

For calculating sensitivity and specificity, BI-RADS 3 and BI-RADS 0 were considered positive findings, which is in accordance with common procedure in screening programs.

For the statistical evaluation, the frequencies of BI-RADS 1 or 2, BI-RADS 3 or 0 , and BI-RADS 4 or 5 were compared for the 3 different evaluations (AV only vs. DBT only vs. AV+DBT). As a measure of agreement (between different methods and readers), kappa statistics were used with intervals of $<0.2,0.21-0.40$, $0.41-0.60,0.6-0.80$, and $0.81-1.0$ defined as poor, fair, moderate, good, and very good agreement, respectively. The $95 \%$ confidence interval (CI) describes the quality of the kappa estimation. Furthermore, the p-value of the $\chi^{2}$ test was used.

\section{Equipment}

The assessment examinations were performed using state-of-the-art digital mammography equipment monitored in the screening program following European guidelines and state-of the-art ultrasound equipment fulfilling national quality assurance standards.

Tomosynthesis was performed using the Siemens MAMMOMAT Inspiration with tomosynthesis option (Siemens $\mathrm{GmbH}$, Erlangen, Germany). This system acquires 25 projections over a tomosynthesis angle of 50 degrees (socalled wide-angle tomosynthesis). The dose was determined by an automatic exposure control which was set up for 1 tomosynthesis scan to 1.5 times the dose of 1 mammographic view. The tomosynthesis slices were reconstructed by the system at $1 \mathrm{~mm}$ slice separation.

Tomosynthesis was applied using 1 single view. We usually chose the view in which the lesion was less obvious on the screening mammogram. In cases with extreme lesion position, the projection was chosen that allowed including the lesion in question.

\section{Reader Training}

The principal investigator (Reader 1) underwent initial training provided by the manufacturer before the study started and had ample experience with contrast-enhanced MRI. Reader 2 had experience with DBT based on $>200$ proven cases.

\section{Results}

Table 1 gives an overview of the histologically proven lesions. The 7 borderline diagnoses were verified by needle biopsy and subsequent surgery.

Table 2 gives an overview of the stages of the primary malignancies. A final pTNM stage is available for $47 / 49$ primary cancers. In 1 patient, a faint lesion of $8 \mathrm{~mm}$ was correctly detected during screening and confirmed by all assessment methods. The patient, however, did not follow the biopsy recommendations until 2 years later when she returned with a lesion of $16 \mathrm{~mm}$ in size (pT1c). Another patient with an invasive breast cancer of $18 \mathrm{~mm}$ in diameter (stage cT1c) un- 
Table 1. Lesions with histopathological proof

\begin{tabular}{llr}
\hline Lesion type & Subtype & $\mathrm{n}$ \\
\hline Malignancy & & 55 \\
& primary & 49 \\
Borderline (B3) & secondary foci & 6 \\
& & 7 \\
& ADH & 3 \\
FEA & radial scar & 2 \\
& & 2 \\
& fibroadenoma & 49 \\
& papilloma (benign, 100\% removed) & 12 \\
& radial scar (very small/removed) & 2 \\
& inflammatory tumor & 1 \\
& lymph node & 1 \\
& benign changes & 32 \\
\hline
\end{tabular}

$\mathrm{ADH}=$ Atypical ductal hyperplasia; FEA= flat epithelial atypia.

Table 2. pT categories of 49 primary malignancies

\begin{tabular}{lc}
\hline Stage & $\mathrm{n}(\%)$ \\
\hline TX & $2(4.1)$ \\
p tis & $7(14.3)$ \\
p T1a and pTmic & $8(16.3)$ \\
p T1b & $15(30.6)$ \\
p T1c & $10(20.4)$ \\
p T 2 & $6(12.2)$ \\
p T3 & $1(2.0)$ \\
\hline
\end{tabular}

Table 3. Overview of the 256 benign and 55 malignant diagnoses established using diagnostic mammography based on screening mammogram and additional views (AV) versus screening mammogram and digital breast tomosynthesis (DBT) versus $\mathrm{AV}+\mathrm{DBT}$

\begin{tabular}{llllllll}
\hline \multirow{2}{*}{ Tests } & \multicolumn{2}{l}{ Benign } & & \multicolumn{3}{l}{ Malignant } \\
\cline { 2 - 3 } \cline { 8 - 9 } & BR1/2 & BR3/0 & BR4/5 & & BR1/2 & BR3/0 & BR4/5 \\
\hline AV & 108 & 58 & 90 & & 5 & 1 & 49 \\
DBT & 145 & 31 & 80 & & 3 & 50 \\
AV+DBT & 139 & 33 & 84 & & 2 & 2 & 51 \\
\hline
\end{tabular}

$\mathrm{BR}=\mathrm{BI}-\mathrm{RADS}$.

derwent neoadjuvant chemotherapy. Surgery after chemotherapy yielded a ypT0 N1a stage with no residual tumor in the breast. The 49 primary malignancies included 7 ductal carcinomas in situ (DCIS), 24 ductal invasive, 13 lobular invasive, 3 tubular, 1 ductulo-lobular, and 1 tubulo-lobular invasive carcinoma (14 G1, 20 G2, 8 G3).

Of the malignancies, $14.3 \%$ were non-invasive (DCIS); $46.9 \%$ of the primary cancers of this study were detected at a size of $<1 \mathrm{~cm}$ (stages 1a or 1b); and 82.5 of the known stages of invasive breast cancers were pT1. The mean size of the detected invasive foci was $13 \mathrm{~mm}$ (median $9 \mathrm{~mm}$ ).

Based on $\mathrm{AV}$, the diagnoses of the 2 readers were identical for 231/311 (74.3\%) lesions. Significantly different judgements (2 BIRADS categories) concerned 9/311 (2.9\%) lesions.

For DBT, 249/311 (80.1\%) diagnoses were identical. Significantly different judgements concerned 8/311 (2.6\%) diagnoses.
Table 4. Sensitivity (SS), specificity (SP), negative (NPV) and positive (PPV) predictive values achieved by additional views (AV) versus digital breast tomosynthesis (DBT) versus $\mathrm{AV}+\mathrm{DBT}$

\begin{tabular}{lllll}
\hline Tests & $\begin{array}{l}\text { SS, } \\
\%\end{array}$ & $\begin{array}{l}\text { SP, } \\
\%\end{array}$ & $\begin{array}{l}\text { NPV, } \\
\%\end{array}$ & $\begin{array}{l}\text { PPV, } \\
\%\end{array}$ \\
\hline AV & 90.9 & 42.2 & 95.6 & 25.3 \\
DBT & 96.4 & 56.6 & 97.4 & 32.3 \\
AV+DBT & 96.4 & 54.3 & 98.6 & 31.2 \\
\hline
\end{tabular}

Table 5. Concordance of digital breast tomosynthesis (DBT) versus additional view (AV) diagnoses in the 49 primary and 6 additional malignant foci ${ }^{\mathrm{a}}$

\begin{tabular}{lllll}
\hline DBT & \multicolumn{2}{l}{ AV } & & \\
\cline { 2 - 4 } & AV BR4/5 & AV BR3/0 & AV BR1/2 & Sum \\
\hline DBT BR4/5 & 48 & 1 & 2 & 51 \\
DBT BR3/0 & 1 & 0 & 1 & 2 \\
DBT BR1/2 & 0 & 0 & 2 & 2 \\
Sum & 49 & 1 & 4 & 55 \\
\hline
\end{tabular}

a 2 foci not visible on DBT or AV (a small DCIS and a 10-mm ILC) were detected by histology and MRI, respectively. 2 foci ( 1 extended DCIS presenting as architectural distortion and 19-mm ILC) were missed on AV and were correctly classified by DBT (BI-RADS 4/5). 1 4-mm IDC (a subtle density) was missed by AV but diagnosed as BI-RADS 0 by DBT. 1 7-mm tubular carcinoma (faint architectural distortion) was more correctly classified by DBT (BI-RADS 4/5) but could not be reproduced by AV (BI-RADS 0). 1 DCIS grade 1 with faint microcalcifications, considered suspicious by AV, was misjudged by DBT and by DBT+AV to probably correspond to calcifications in small tortured vessels (BI-RADS 3).

$\mathrm{BR}=\mathrm{BI}-\mathrm{RADS}$; DCIS $=$ ductal carcinoma in situ; $\mathrm{ILC}=\mathrm{IDC}=$ invasive lobular carcinoma; IDC = invasive ductal carcinoma.

Table 6. Concordance of digital breast tomosynthesis (DBT) versus additional view (AV) diagnoses in the 256 benign lesions (BR = BI-RADS)

\begin{tabular}{lllll}
\hline DBT & \multicolumn{1}{l}{ AV } & & & \\
\cline { 2 - 4 } & AV BR4/5 & AV BR3/0 & AV BR1/2 & Sum \\
\hline DBT BR4/5 & 69 & 7 & 4 & 80 \\
DBT BR3/0 & 7 & 19 & 5 & 31 \\
DBT BR1/2 & 14 & 32 & 99 & 145 \\
Sum & 90 & 58 & 108 & 256 \\
\hline
\end{tabular}

For DBT+AV, 235/311 (75.6\%) diagnoses were identical. Significantly different judgements concerned $2 / 311$ (0.7\%) diagnoses.

Table 3 shows the BI-RADS classifications of the 256 benign lesions (including 7 borderline lesions) and the 55 malignant lesions. Table 4 shows the corresponding sensitivities and specificities.

Statistically, the 3 methods differed significantly concerning the BI-RADS classifications assigned to benign and malignant lesions $(\mathrm{p}<0.05)$. Accordingly, agreement between the methods was moderate for malignancies (50/55 $=90.9 \%$; kappa: 0.46 ; $95 \%$ CI 0.02 , $0.91 ; \mathrm{p}=0.059)$ and for benign lesions (187/256 = 73\%; kappa: 0.57; CI 0.49, 0.67; $\mathrm{p}<0.001$ ).

As shown in tables 5 and 6 , there were small differences concerning the accuracy of detection and classification of malignancy. 


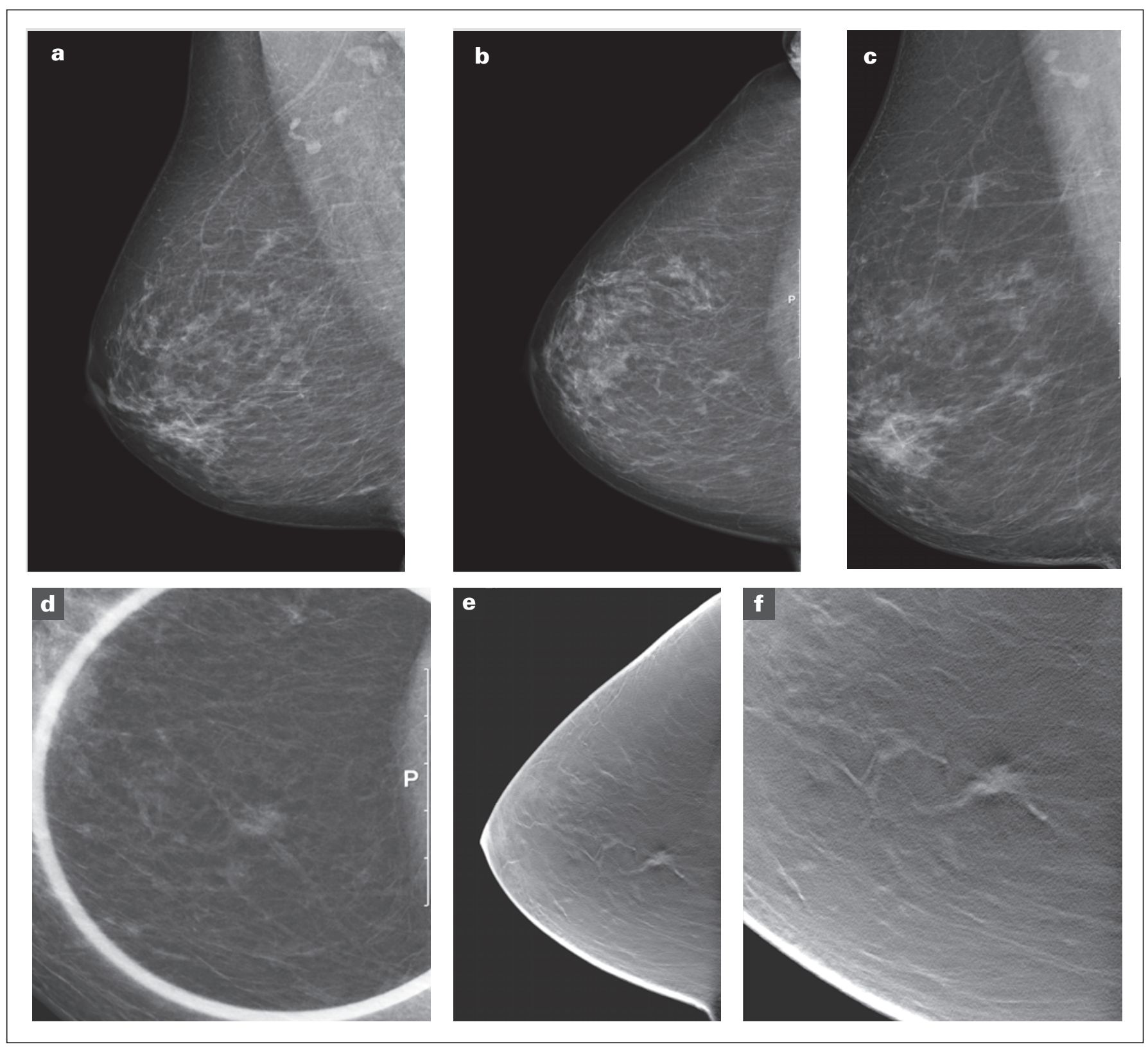

Fig. 1. First screening mammogram in a 58 -year-old woman who was invited for further assessment of a medial focal asymmetry in her right breast. a, b Screening mammography (right MLO and CC views). c, d Additional views (RML and CC spot view). Based on the existing images, the focal asymmetry probably correlated with a slight cranial asymmetry on the RML view. However, there were multiple further islands of breast tissue. Ultrasound did not show a correlating lesion. Since there was no architectural distortion and the slight asymmetry contained fatty inclusions, the lesion might have been misclassified as a probably benign lesion. e, $\mathbf{f}$ Tomosynthesis clearly demonstrates a lesion in the upper cuts of the right breast. The lesion appears quite dense and contains fine spiculations. Stereotactic vacuum-assisted breast biopsy under mammography guidance was performed, followed by breast-conserving therapy. Histology confirmed a pT1b pN0 G2 ductal breast cancer.

Sensitivity was highest for DBT+AV, followed by DBT and by AV. The differences were not statistically significant.

BI-RADS classifications assigned to benign lesions differed significantly for the 3 methods ( $\mathrm{p}<0.01$ ), as did specificities ( $\mathrm{p}$ $<0.001$ ). The number of correctly classified benign lesions was highest for DBT, followed by DBT+AV. It was lowest for AV alone.

The differences in specificity were significant comparing DBT versus AV $(p<0.001)$ and DBT+AV versus AV $(p=0.004)$. Only $\mathrm{DTB}$ and $\mathrm{DBT}+\mathrm{AV}$ were not different in pairwise comparison.
Overall, both DBT and DBT+AV proved at least equivalent to $\mathrm{AV}$. In a retrospective evaluation, we considered $88.8 \%$ of the 517 spot views as unnecessary for making a correct diagnosis.

The highest gain through using DBT was achieved for differentiation architectural distortions and densities, followed by masses. No diagnostic gain was noted for global asymmetries and microcalcifications. Additional information was gained in breasts of any density (ACR1-4). The lowest gain was noted in breasts with an ACR4 density, the highest for densities of ACR2 and 3. 
In this study, 31 (10\%) abnormalities were visible on 1 view only and could only be correctly localized by DBT. This concerned 23/256 (9\%) benign lesions and 8/55 (14.5\%) malignant lesions. 6 of these 8 malignancies were also not visible on ultrasound.

Even though the main purpose of this study did not concern ultrasound, we retrospectively analyzed the added information from ultrasound for the 55 cancer cases. Altogether, 23/55 malignant lesions could not be detected by ultrasound even in retrospect. They included 13 invasive breast cancers ( 2 densities, 2 cases with microcalcifications only, 3 masses, 6 architectural distortions) and 10 DCIS (8 cases of microcalcifications, 1 density, 1 architectural distortion). No cancer of this series was detected by ultrasound only.

Figure 1 demonstrates the different information available from $\mathrm{AV}$ versus DBT.

\section{Discussion}

In this study, the value of wide-angle DBT for imaging assessment of screen-detected abnormalities was investigated comparing 3 combinations: screening mammography plus AV, screening mammography plus DBT, and screening mammography plus $\mathrm{AV}+\mathrm{DBT}$. According to our results, DBT proved at least equivalent to $\mathrm{AV}$ in the assessment of screen-detected abnormalities.

The fact that only single foci were additionally detected by DBT, yielding no statistically significant difference in sensitivity compared to $\mathrm{AV}$, is an expected result for this design, since only 285 women were examined and the expected incidence of mammographically occult cancers is $<2 / 1,000$.

Even with this design, DBT appeared slightly more sensitive than AV. However, there were cases in which DBT had advantages and vice versa. Hence, unless the DBT information is clear, information from AV might still be valuable in individual cases.

For specificity, significantly better results were achieved comparing DBT versus $\mathrm{AV}$ and comparing $\mathrm{DBT}+\mathrm{AV}$ versus $\mathrm{AV}$. The use of DBT for lesion assessment thus promises to reduce both histopathological assessments and short-term follow-up examinations as well as patient distress and costs while improving diagnostic accuracy.

Provided that our results can be reproduced prospectively and $88 \%$ of AV can be spared, the overall radiation dose of assessment with DBT (using 1.5-times the dosage of a mammographic view) would remain more or less unchanged (compared to formerly 1.8 AV on average). Further dose savings appear possible by reducing stereotactic biopsies and short-term follow-up.

The highest diagnostic gain was noted in breasts with ACR2 and 3 , but some gain was possible in all density ranges. DBT was most helpful for architectural distortions and densities but did not contribute significant information over AV for microcalcifications or for the few cases of global asymmetry.

The above observations agree with the results of most other studies [3-11]. Our different experience regarding microcalcifications as compared to Kopans et al. [12] may be explained by differ- ences in study design. We compared magnification views (not screening mammograms) with DBT. According to our results, (non-magnified) DBT should generally not be used instead of magnification views for assessing screen-detected microcalcifications.

In addition to the results concerning diagnostic accuracy, the capability of exactly localizing a lesion that is seen as a subtle (and often questionable) abnormality on 1 view only has proven very valuable. It may aid both the differentiation between lesions and superimpositions and the planning of interventions.

This study was designed to only compare the mammographic techniques (AV vs. DBT vs. AV+DBT). Ultrasound information was not included in the comparison, since it was not assumed that i) ultrasound should replace a mammographic technique (due to its limited sensitivity for very small mammographically detected lesions), and ii) a mammographic technique could substitute the complimentary information available from ultrasound.

Retrospectively, this decision is also supported by the fact that in this study 23/55 mammographically detected malignancies were not visible on ultrasound. This high percentage of lesions only made visible by mammographic techniques may be due to the overall small size of the lesions and the selection of lesions requiring mammographic AV. Thus, an important part of the additional information contributed by DBT in this study is indispensable and could not be provided by ultrasound.

Since ultrasound information was available for the non-blinded interpretation of $\mathrm{AV}, \mathrm{DBT}$, as well as $\mathrm{DBT}+\mathrm{AV}$, we do not expect that its presence or absence in this design introduced a significant bias in the comparative evaluation.

The major drawback of our study, which included a prospective and a retrospective reading, concerns the fact that the prospective diagnoses (by the first reader) were made in a non-blinded fashion with regard to DBT, AV, and ultrasound. However, the reading was based on a realistic situation as it would arise during screening assessment.

The second reading (by Reader 2) was a retrospective reading of the anonymized cases, and the second reader was blinded to the diagnoses of the first reader, and both were blinded to the final results.

For the consensus reading, which took place 3 years after the study had ended, recency bias (by recalling single cancer cases) cannot be completely excluded. However, the number of cancers considered positive in the consensus meeting did not differ from the number of cancers considered positive by Reader 2. Recall of negative cases is improbable. Being aware of potential bias, special care was taken throughout the study to rate methods differently only if the information clearly differed.

Overall, the lesion selection was representative of consecutive screen-detected abnormalities in our screening unit requiring assessment by AV. In retrospect, however, we noted some 'under-recruitment' of cases with microcalcifications. We explain this by a certain bias of the team which probably occurred during recruitment, in that the team was less convinced of the reliability of the DBT results in relation to microcalcifications compared to other indications. This lack of faith may also have affected the patients' 
decision for or against participation. Hence, for cases with microcalcifications, absence of selection bias can in retrospect not be warranted. This relative under-recruitment explains the somewhat lower proportion of DCIS in this study (14.3\%, as compared to $21 \%$ on average in our screening unit).

In spite of the above limitations, we consider it important to communicate the data we have available.

According to our knowledge, this is one of few large case series where DBT was offered consecutively to women undergoing assessment for a screen-detected abnormality. It is the only study in which DBT was compared with DBT+AV and with AV only, and it is one of the very few studies with long-term follow-up of benign lesions.

A similar population was examined by Tagliafico et al. [7]; however, the number of cases was much smaller. The studies by Cornford et al. [9] and Morel et al. [10] included > 300 patients; however, different equipment was used.

The study by Morel et al. [10] was based on a retrospective reading design. Each study was read by only 1 of 7 readers. Since the readings systematically started with $\mathrm{AV}$, followed 3 weeks later by the DBT reading performed by the same reader, a certain bias in favor of DBT cannot be excluded.

Cornford et al. [9] were evidently aware of the advantages and disadvantages of both prospective and retrospective readings. Hence, they separately evaluated both situations, i.e. a prospective consensus reading (during assessment) and a retrospective reading (without subsequent consensus). The authors observed no significant differences between the prospective and the retrospective reading, which is in agreement with our experience.

While this paper is representative of the underlying study, some of the presented cases were also used in 2 subsequent multi-reader studies. One multi-reader study has just been published [13]. It showed equivalence of DBT versus AV and only slightly improved accuracy. Compared to this study, the above described potential bias can be excluded. However, some bias against DBT may have occurred, since the region of interest by nature was obvious on the spot views, while it was unknown on the DBT image set.

In summary, so far, all investigations confirm non-inferiority of DBT versus AV when used for lesions that are not classified as ' $m i-$ crocalcifications only'. Our data, which are based on a large number of consecutive screen-detected abnormalities, confirm these results. According to our experience, DBT promises higher specificity without loss of sensitivity.

Based on the existing data, we consider the use of DBT in the diagnostic setting and assessment of screen-detected lesions justified. Further studies are desirable to improve the present knowledge on the reproducibility of the first results.

\section{Disclosure Statement}

Sylvia Heywang-Köbrunner has a research cooperation concerning tomosynthesis (cooperation with Siemens). The payment compensates for the work delivered in the study and a cooperation concerning training on tomosynthesis and intervention (cooperation with Medicor/Hologic). The payment compensates for training courses and presentations on the above subject. Further she received compensation for travel (and sometimes for absence from work) for invited presentations given at regional, national or international meetings (different organizers) and for consulting official institutions (insurances, government institutions). Overall these payments do not compensate the costs. Thomas Mertelmeier is employee of Siemens Healthcare GmbH, Erlangen, Germany and Stockholder of Siemens AG. The other authors declare that no conflicts of interest exists according to the guidelines of the International Committee of Medical Journal Editors.

\section{References}

1 Duijm LE, Groenewoud JH, de Koning HJ, et al.: Delayed diagnosis of breast cancer in women recalled for suspicious screening mammography. Eur J Cancer 2009;45:774-781.

2 Von Euler-Chelpin M, Kuchiki M, Vejborg I: Increased risk of breast cancer in women with false-positive test: the role of misclassification. Cancer Epidemiol 2014;38:619-622.

3 Teertstra HJ, Loo CE, van den Bosch MA, et al.: Breast tomosynthesis in clinical practice: initial results. Eur Radiol 2010;20:16-24.

4 Skaane P, Gullien R, Bjørndal H, et al.: Digital breast tomosynthesis (DBT): initial experience in a clinical setting. Acta Radiol 2012;53:524-529.

5 Noroozian M, Hadjiiski L, Rahnama-Moghadam S, et al.: Digital breast tomosynthesis is comparable to mammographic spot views for mass characterization. Radiology 2012;262:61-68.
6 Zuley ML, Bandos AI, Ganott MA, et al.: Digital breast tomosynthesis versus supplemental diagnostic mammographic views for evaluation of noncalcified breast lesions. Radiology 2013;266:89-95.

7 Tagliafico, Astengo D, Cavagnetto F, et al.: One-to-one comparison between digital spot compression view and digital breast tomosynthesis. Eur Radiol 2012;22: 539-544.

8 Brandt KR, Craig DA, Hoskins TL, et al.: Can digital breast tomosynthesis replace conventional diagnostic mammography views for screening recalls without calcifications? A comparison study in a simulated clinical setting. AJR Am J Roentgenol 2013;200:291-298.

9 Cornford EJ, Turnbull AE, James JJ, et al.: Accuracy of GE digital breast tomosynthesis vs supplementary mammographic views for diagnosis of screen-detected soft-tissue breast lesions. Br J Radiol 2016;89:20150735.
10 Morel JC, Iqbal A, Wasan RK, et al.: The accuracy of digital breast tomosynthesis compared with coned compression magnification mammography in the assessment of abnormalities found on mammography. Clin Radiol 2014;69:1112-1116.

11 Spangler ML, Zuley ML, Sumkin JH, et al.: Detection and classification of calcifications on digital breast tomosynthesis and 2D digital mammography: a comparison. AJR Am J Roentgenol 2011;196:320-324.

12 Kopans D, Gavenonis S, Halpern E, Moore R: Calcifications in the breast and digital breast tomosynthesis. Breast J 2011;17:638-644.

13 Whelehan P, Heywang-Köbrunner SH, Vinnicombe SJ, et al.: Clinical performance of Siemens digital breast tomosynthesis versus standard supplementary mammography for the assessment of screen-detected softtissue abnormalities: a multi-reader study. Clin Radiol 2017;72:95.e9-95.e15. 